\title{
Identification and validation of tumor microenvironment-related genes of prognostic value in lung adenocarcinoma
}

\author{
JINGYAN YUAN, BO YUAN, LIZHONG ZENG, BOXUAN LIU, YANG CHEN, \\ XIA MENG, RUIYING SUN, XIN LV, WEI WANG and SHUANYING YANG \\ Department of Respiratory and Critical Care Medicine, The Second Affiliated Hospital, \\ Xi'an Jiaotong University, Xi'an, Shaanxi 710004, P.R. China
}

Received September 29, 2019; Accepted March 17, 2020

DOI: $10.3892 / \mathrm{ol} .2020 .11735$

\begin{abstract}
Lung adenocarcinoma (LUAD) is a major subtype of non-small cell lung cancer. Despite significant progress in its diagnosis and treatment, the mortality and morbidity rate of LUAD remains high worldwide. The aim of the present study was to perform a systematic investigation of the tumor microenvironment (TME) and identify TME-related genes of prognostic value in patients with LUAD. Firstly, the immune scores and stromal scores of patients with LUAD from The Cancer Genome Atlas were calculated using the Estimation of STromal and Immune cells in MAlignant Tumors using Expression data algorithm, and a total of 281 prognostic TME-related genes were identified. Subsequently, functional analysis and protein-protein interaction network analysis revealed that these genes were mainly related to immune response, inflammatory response and chemotaxis. Finally, two independent LUAD cohorts from the Gene Expression Omnibus database were used to validate these genes, and 4 genes (GTPase IMAP family member 1, T-cell surface glycoprotein $\mathrm{CD} 1 \mathrm{~b}$, integrin alpha-L and leukocyte surface antigen CD53) were identified, and downregulation of these genes was indicated to be associated with poor overall survival rate in patients with LUAD. In conclusion, a comprehensive analysis of TME was performed and 4 prognostic TME-related genes in patients with LUAD were identified.
\end{abstract}

\section{Introduction}

Lung adenocarcinoma (LUAD) is a subtype of non-small cell lung cancer (NSCLC) and represents the most common histological type of NSCLC (1). Although significant progress has

Correspondence to: Dr Shuanying Yang, Department of Respiratory and Critical Care Medicine, The Second Affiliated Hospital, Xi'an Jiaotong University, 157 Xiwu Road, Xi'an, Shaanxi 710004, P.R. China

E-mail: yangshuanying66@163.com

Key words: The Cancer Genome Atlas, Gene Expression Omnibus, lung adenocarcinoma, tumor microenvironment, survival analysis been made in the treatment of LUAD with the use of molecular targeted therapies, the 5-year survival rate of patients with LUAD is still $<20 \%$ (2). Therefore, novel biomarkers are urgently required for significant improvements in prognosis. Progress has been made in the application of immunotherapy in the treatment of LUAD. For example, the application of programmed cell death-1 (PD-1) inhibitors, PD-ligand 1 inhibitors and cytotoxic T-lymphocyte-associated protein 4 (CTLA-4) inhibitors have been shown to enhance intratumoral immune responses and improve prognosis in patients with LUAD in numerous preclinical or clinical studies (3-6). However, some studies have also identified the limitations of immunotherapy, including severe adverse effects and low response in some patients (7). One of the factors which diminishes the efficacy of tumor immunotherapy is the tumor microenvironment (TME) $(8,9)$, which is heterogeneous in composition and contains cellular components, growth factors, proteases and extracellular matrix (10). As a result, it is particularly important to identify TME-related biomarkers in order to identify patients who will have an improved prognosis after receiving immunotherapy.

The TME regulates major hallmarks of cancer, including angiogenesis, inflammation, immune suppression, epithelial-mesenchymal transition and metastasis (11). Previous studies have demonstrated that immune-inflamed TMEs express high levels of cytotoxic lymphocytes as well as immune activation markers, and tumor purity is decreased $(12,13)$. Tumors with this type of TMEs are often associated with a favorable prognosis (14), however, patients with immune-excluded TMEs or a high tumor purity have a poorer survival instead. In addition, Givechian et al (15) reported that patients with immune-inflamed LUAD were associated with improved overall survival (OS) compared with patients with immune-excluded LUAD. Behind this phenomenon, genes such as CD8 and PRF1 (12,15-17) or signaling pathways such as ribosomal, metabolic and chemokine signaling pathways $(15,18)$ may serve an important role. Therefore, recognizing these genes and utilizing them provides a deep understanding of TME in patients with LUAD, which could guide immunotherapy. With the development of bioinformatics, some algorithms have been applied to evaluate the tumor purity of TME according to the specific gene expression signature of immune or/and stromal cells $(19,20)$. In 2013, 
Yoshihara et al (20) invented an algorithm termed Estimation of STromal and Immune cells in MAlignant Tumors using Expression data (ESTIMATE) to analyze stromal and immune cells that form the major non-tumor constituents of tumor samples. This algorithm calculates the stromal and immune scores to predict the tumor purity of tumor tissues. In the present study, The Cancer Genome Atlas (TCGA) database and ESTIMATE algorithm were utilized to identify TME-related genes to predict outcomes in patients with LUAD.

\section{Materials and methods}

Database. Level 3 gene expression data for 517 patients with LUAD was downloaded from TCGA data portal (https://tcga-data.nci.nih.gov/tcga/) and was analyzed using the Illumine Hiseq 2000 RNA Sequencing v.2 platform [University of North Carolina (UCSC) TCGA genome characterization center; October 10, 2017] and RNA sequencing data was downloaded from UCSC Xena browser (https://xena. ucsc.edu/). Clinical data, including age, sex, histological type, tumor metastasis conditions, epidermal growth factor receptor (EGFR) mutation status, overall survival time and outcome were downloaded from TCGA data portal. Immune and stromal scores of 517 patients with LUAD were calculated by applying the ESTIMATE algorithm to the downloaded dataset. For validation, the Gene Expression Omnibus (GEO) database was used to compare gene expression profiling of patients with LUAD with clinical data of survival and outcome. Finally, two independent datasets, GES37745 (n=106) (21) and GES29013 ( $n=31$ ) (22), were used to validate the identified genes.

Identification of differentially expressed genes (DEGs). Based on the ESTIMATE results, all samples were divided into high/low immune-score groups and high/low stromal-score groups to select intersection genes. The cut-off value of identifying high immune score group or low immune score group was 980.35. The cut-off value for identifying high stromal score group or low stromal score group was 36.85. DEG data analysis was performed using limma package (23). The cut-off values for screening DEGs were set as fold change $(\mathrm{FC})>2$ or $<-2$ and $\mathrm{P}<0.05$. Volcano plots were generated using the ggplot2 package in R software v.3.5 (24).

Construction of protein-protein interaction (PPI) network. The Search Tool for the Retrieval of Interacting Genes (STRING) online database was used to analyze the PPI network of DEGs (25). The DEGs were uploaded to the STRING online website and the interactive relationships were determined. The cut-off value of the minimum required interaction score was set as 0.700. Subsequently, the Cytoscape software v.3.6 (26) was used to construct the PPI network and Molecular Complex Detection (MCODE) was used to identify the top three complete module clusters (26).

Functional analysis of DEGs. The Database for Annotation, Visualization and Integrated Discovery (DAVID) website was used to perform Gene Ontology (GO) analysis and Kyoto Encyclopedia of Genes and Genomes (KEGG) analysis and the false discovery rate $<0.05$ was defined as the cut-off value (27).
Statistical analysis. All data are presented expressed as the mean \pm standard deviation. Student's t-test (two groups) and one-way ANOVA (multiple groups) were used to compare the immune and stromal scores in different groups using Graph-Pad Prism v7.0 software. (GraphPad Software, Inc.). The post hoc test used following ANOVA was Tukey's multiple comparisons test. OS curves were created using the Kaplan-Meier survival analysis and estimated using two-sided log rank test. Differential analysis of expressed genes and volcano plots was performed using $\mathrm{R}$ software v.3.5. $\mathrm{P}<0.05$ was considered to indicate a statistically significant difference.

\section{Results}

Stromal and immune scores are significantly associated with TNM stage, epidermal growth factor receptor mutation status, distant metastasis and clinical outcome in patients with $L U A D$. To determine the relationship between ESTIMATE scores and clinical characteristics, the gene expression data as well as clinical information of 517 patients with LUAD were downloaded from the TCGA database. The dataset included patients in which initial pathological diagnosis was made between 1991 and 2013. Among these patients, 277 cases $(54 \%)$ were male and 240 cases $(46 \%)$ were female. The immune and stromal scores of each patient were determined, the stromal scores ranged from $-1,959.31$ to $2,098.77$, and the immune scores ranged from $-1,355.85$ to $3,286.67$. As presented in Fig. 1A, the immune scores of each TNM stage were compared. The average immune scores of patients with stage I were the highest amongst all of the TNM stages, followed by that of stage II, and stage III. Patients with stage IV had the lowest immune scores (Fig. 1A; $\mathrm{P}=0.0305$, one-way ANOVA). Fig. 1B revealed the stromal scores of each TNM stage. The stromal scores were highest in patients with stage II LUAD, followed by stage I, stage III and stage IV (Fig. 1B; P=0.1111).

Subsequently, the immune and stromal scores were compared between the distant metastasis group and the non-distant metastasis group. The average immune and stromal scores of the distant metastasis group were significantly lower compared with that in the non-distant metastasis group (Fig. 1C and D). The immune and stromal scores between lymph node metastasis group and non-lymph node metastasis were also compared, however, the results revealed there was no statistically significant difference between the two groups (Fig. S1A and B).

To the best of our knowledge, the frequency of EGFR gene mutation among Asian populations with NSCLC is $230 \%$ (28). In Fig. $1 \mathrm{E}$ and $\mathrm{F}$, the average immune and stromal scores in the EGFR mutant group and the EGFR wild-type group were shown. The stromal score of the EGFR mutant group was significantly higher compared with that of the EGFR wild-type group $(\mathrm{P}<0.0001)$, while the immune score of the EGFR mutant group was also higher compared with that of the EGFR wild-type group, although the result was not statistically significant $(\mathrm{P}=0.5113)$.

To determine the association between OS and immune and stromal scores, patients were divided into two groups based on high or low scores using median value and Kaplan-Meier survival curves were subsequently plotted. As indicated by Fig. 1G, the median OS time of the low immune score group was significantly 
A

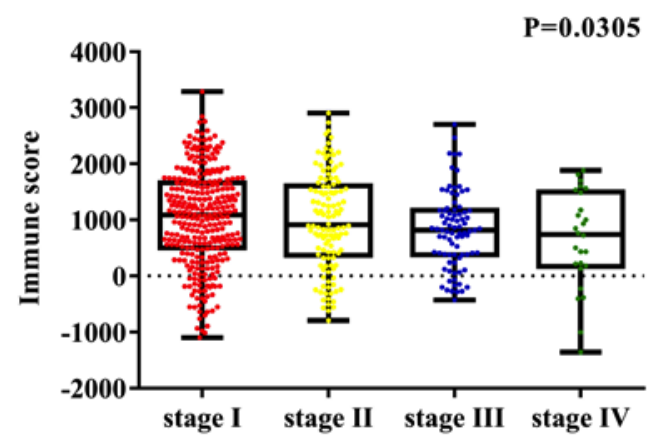

C

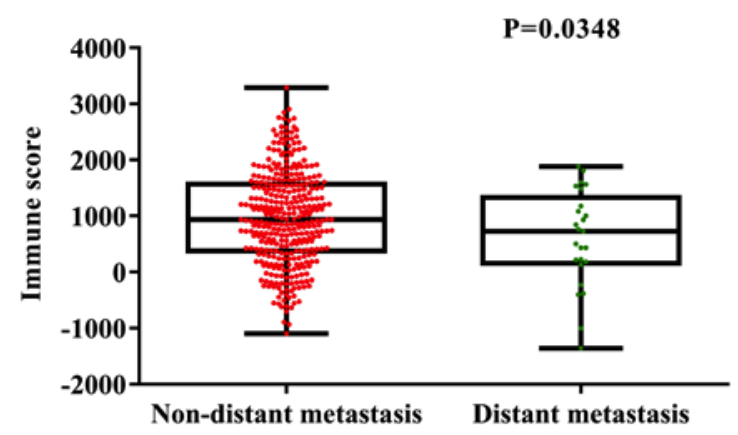

E

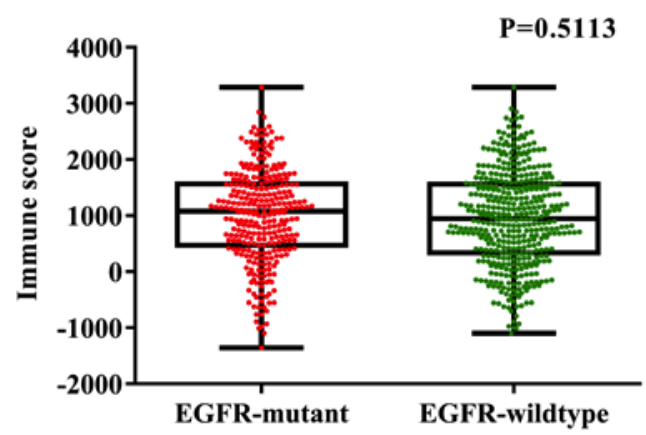

G

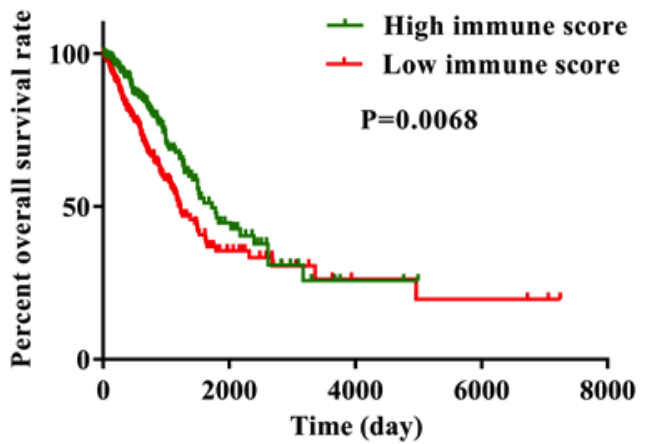

B

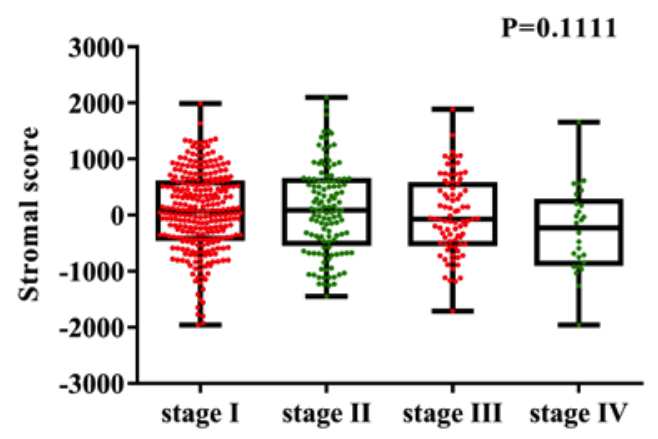

D

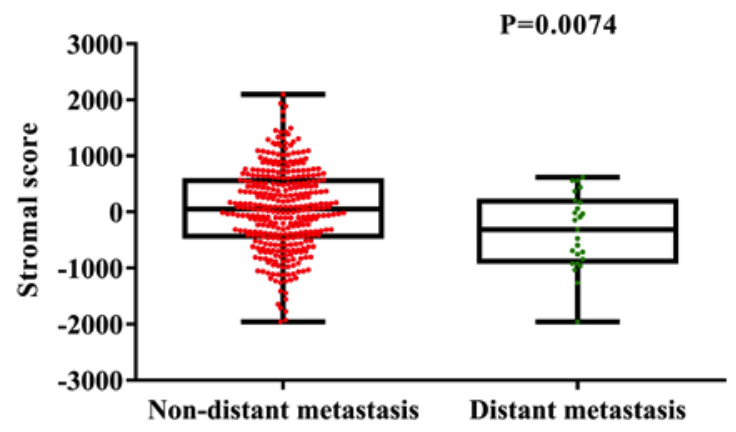

$\mathrm{F}$

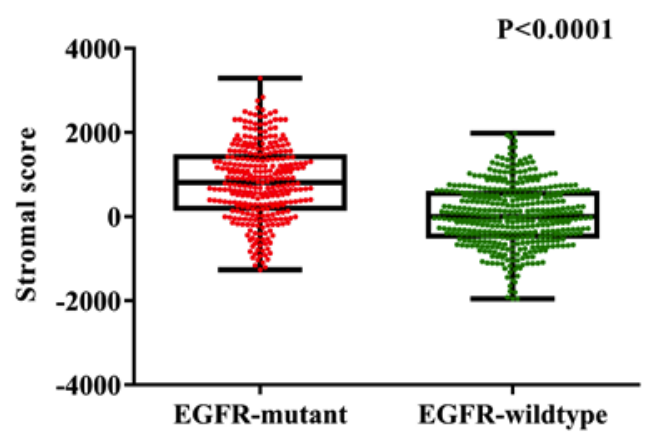

$\mathrm{H}$

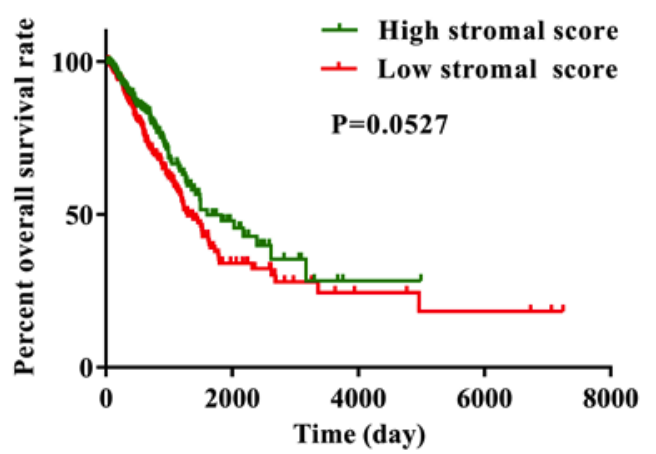

Figure 1. Immune and stromal scores are significantly associated with TNM stage, EGFR mutation status, distant metastasis and clinical outcome in patients with LUAD. Distribution of (A) immune and (B) stromal scores of each TNM stage. Distribution of (C) immune and (D) stomal scores for distant metastasis and non-distant metastasis LUAD cases. Distribution of (E) immune and (F) stromal scores for EGFR wide-type and EGFR mutant LUAD cases. Analysis of patients' overall survival based on $(\mathrm{G})$ immune and $(\mathrm{H})$ stromal scores. EGFR, epidermal growth factor receptor; LUAD, lung adenocarcinoma.

shorter compared with that of the high immune score group $(1,229$ vs. 1,725 days; $\mathrm{P}=0.0068$ using the log-rank test). As shown in Fig. $1 \mathrm{H}$, the median OS time of the low stromal score group was shorter compared with that of the high stromal score group $(1,293$ vs. 1,600 days; $\mathrm{P}=0.0527$; log-rank test), although there were no statistically significant differences between the two groups. 
A
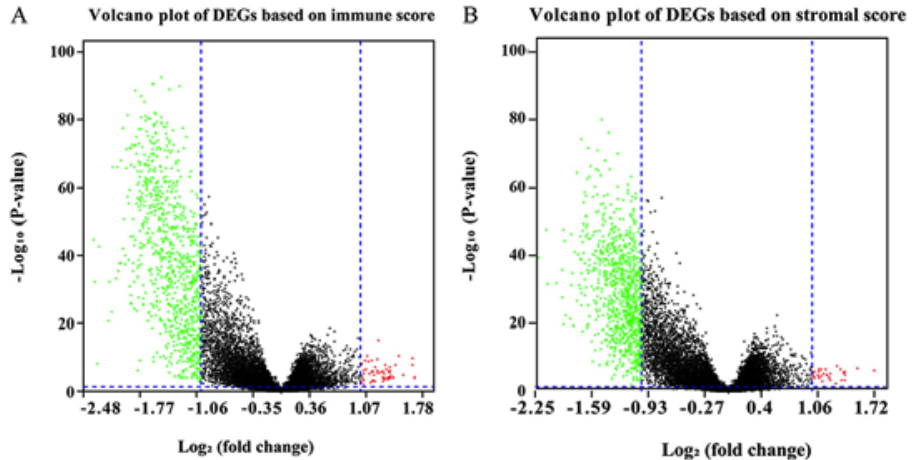
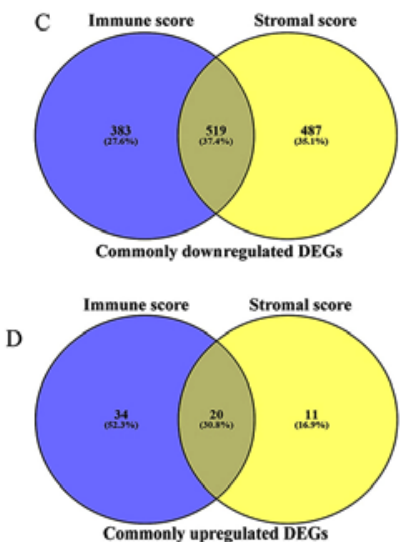

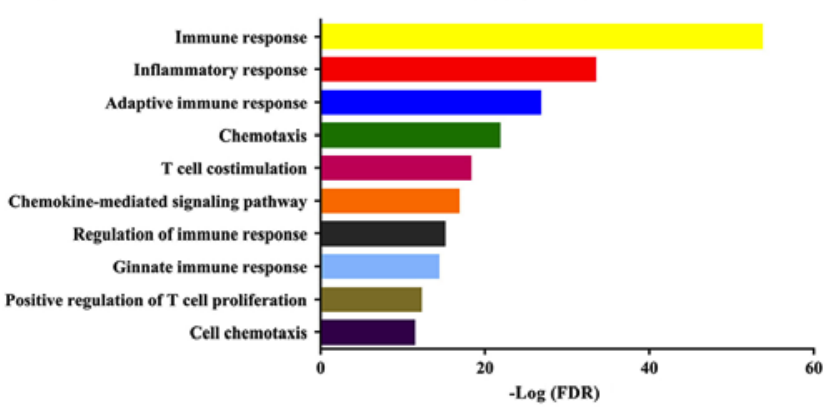

G

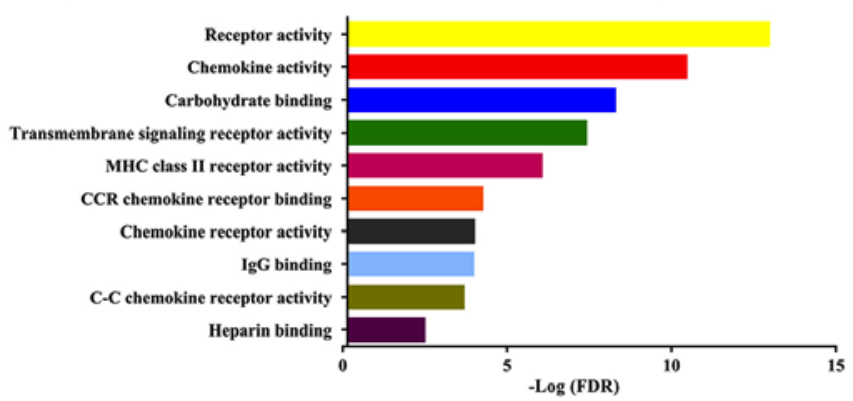

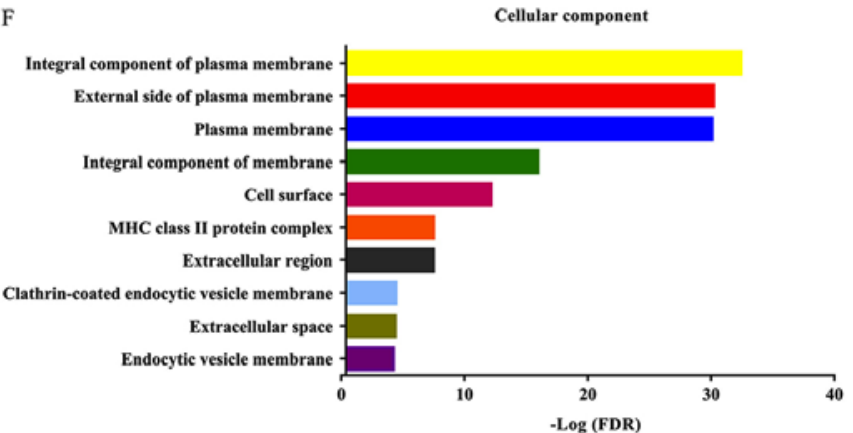

H

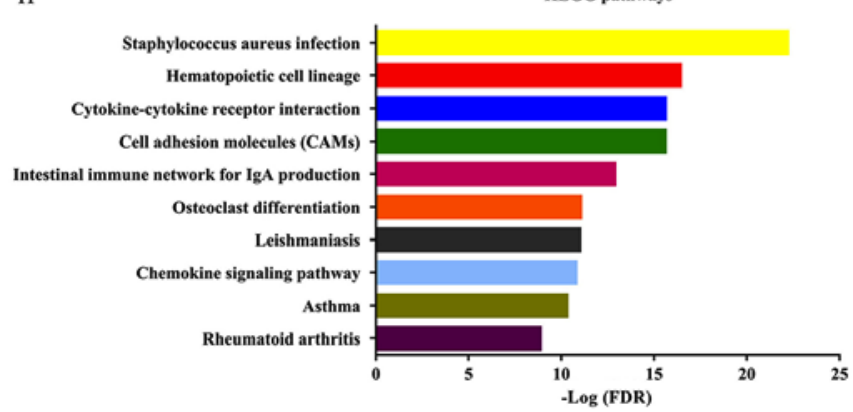

Figure 2. Identification of DEGs in high and low immune and stromal score groups. (A) Volcano plot of DEGs based on immune scores of low score group vs. high score group. (B) Volcano plot of DEGs based on stromal scores of low score group vs. high score group. Venn diagram analysis of (C) common downregulated DEGs and (D) common upregulated DEGs. Top ten (E) Biological process, (F) Cellular component and (G) Molecular function, Gene Ontology terms of common downregulated DEGs. (H) Top ten KEGG pathways of common downregulated DEGs. DEGs, differentially expressed genes; KEGG, Kyoto Encyclopedia of Genes and Genomes; FDR, false discovery rate.

Identification of DEGs of immune and stromal score groups. The gene expression matrix file of 517 patients with LUAD downloaded from TCGA database was used to assess the gene expression profile differences between high immune/stromal scores and low immune/stromal scores. As shown in Fig. 2A and B, volcano plots revealed the DEG profile of low immune/stromal score groups compared with high immune/stromal score groups. A total of 902 downregulated genes and 54 upregulated genes were identified in the immune score group (low vs. high, $\mathrm{FC}>2$ or $<-2 ; \mathrm{P}<0.05$ ). Similarly, a total of 1,006 downregulated genes and 31 upregulated genes were identified in the stromal score group (low vs. high; FC $>2$ or $<-2 ; \mathrm{P}<0.05)$. Furthermore, 519 downregulated DEGs as well as 20 upregulated DEGs were found in both the immune and stromal groups (Fig. 2C and D; Table SI). Only the 519 intersection downregulated genes were included in the further analysis. The 20 intersection upregulated DEGs were not included due to being the minority of common DEGs. Furthermore, the current analysis was primary focused on downregulated genes, the overexpression of which might become a target for future gene therapy. The prognostic value of the 20 upregulated genes was performed (Table SII) and the results revealed that the hazard ratio (HR) of these genes was $\sim 1$, and the P-value of most of these genes was $>0.05$. The 519 DEGs were used for all subsequent analysis. The DAVID online tool was used to determine the potential functions of these genes. Fig. $2 \mathrm{E}-\mathrm{G}$ revealed the top $\mathrm{GO}$ terms of these genes. These genes were involved in biological processes (BPs), including 'immune response', 'inflammatory response' and 'chemotaxis' (Fig. 2E). The molecular functions (MFs) of these genes included 'receptor activity', 'chemokine activity' and 'transmembrane signaling receptor activity’ (Fig. 2G). Cellular components (CCs) of these genes included integral component of plasma membrane, 'external 

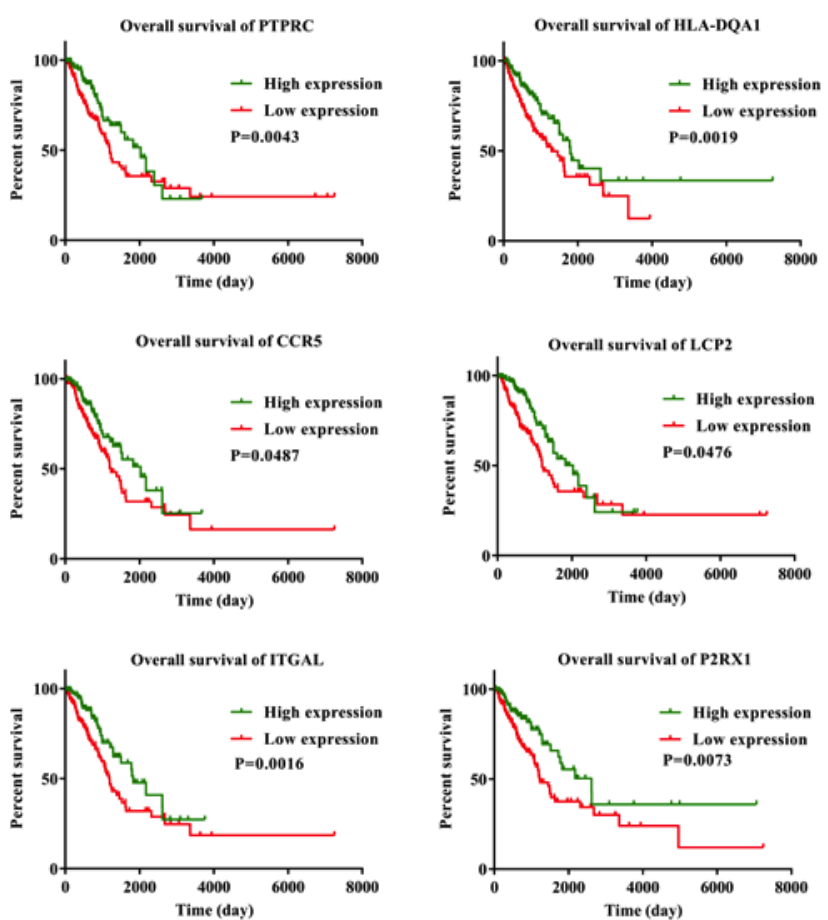
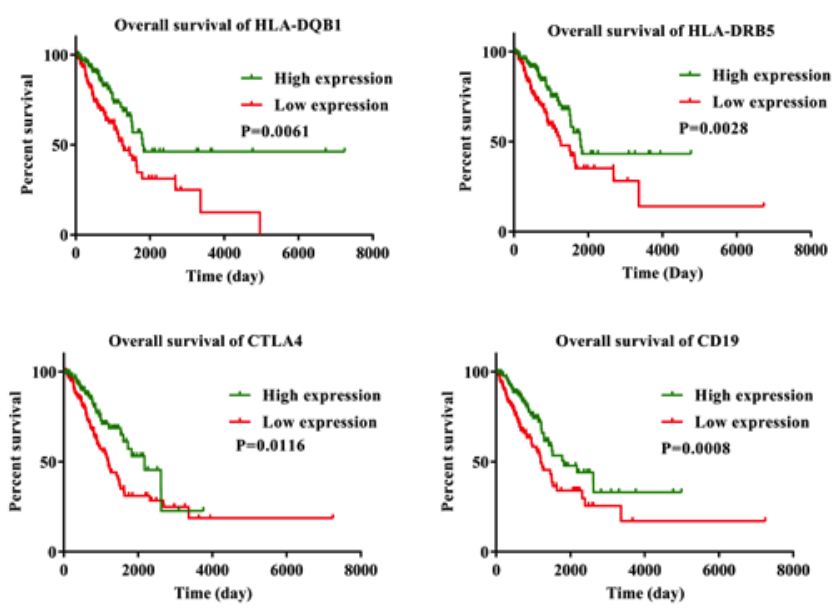

Figure 3. Kaplan-Meier survival curves for some of the DEGs associated with overall survival.

side of plasma membrane' and 'plasma membrane' (Fig. 2F). The potential pathways these genes may be associated with were also investigated using DAVID. The top 10 KEGG pathways these genes are involved in included 'Staphylococcus aureus infection', 'cytokine-cytokine receptor interaction', 'cell adhesion molecules (CAMs)' and 'chemokine signaling pathway'.

Prognostic value analysis of 519 common downregulated DEGs. To determine if the 519 common downregulated genes were associated with OS, Kaplan-Meier survival curves were plotted. A total of 281 genes were identified to be associated with favorable OS using log-rank test $(\mathrm{P}<0.05 ; \mathrm{HR}<1$; Table SIII). Kaplan-Meier survival curves for some of the genes are presented in Fig. 3. Kaplan-Meier survival curves for 10 representative genes were selected and these 10 genes were found to have multiple connections with other genes (Fig. 4).

PPI network construction of 281 DEGs of prognostic value. The PPI network using STRING online tool was used to identify the interaction among the identified DEGs. This network was comprised of 273 nodes and 580 edges and Cytoscape software was used to visualize the network (Fig. 4A). The top three modules of this PPI network were selected using the MCODE plugin. The first module (Fig. 4B) contained 26 nodes and 162 edges, and protein tyrosine phosphatase receptor type $\mathrm{C}$, lymphocyte cytosolic protein 2 , HLA class II histocompatibility antigen DR beta 5 chain, HLA class II histocompatibility antigen DQ alpha 1 chain, HLA class II histocompatibility antigen DQ beta 1 chain and C-C chemokine receptor type 5 were of interest as they were found to have multiple connections with other genes. The second module (Fig. 4C) contained seven nodes and 21 edges, identifying CTLA-4 and B-lymphocyte antigen CD19 as the most connected genes. The third module (Fig. 4D) was formed by 11 nodes and 32 edges, and integrin alpha-L (ITGAL) and $\mathrm{P} 2 \mathrm{X}$ purinoceptor 1 had the highest connectivity degree values.

Functional analysis of genes of prognostic value. GO and KEGG pathway analysis of genes of prognostic value was subsequently performed. Fig. 5A shows the BPs in which these genes are involved, and the top 10 terms were identified including 'immune response', 'T cell receptor signaling pathway', 'adaptive immune response', and 'regulation of immune response'. Fig. 5B shows the top $10 \mathrm{CC}$ terms, including 'external side of membrane', 'MHC class II protein complex' and plasma membrane. Fig. 5C shows the top 10 significant $\mathrm{MF}$ terms of these genes, which were 'transmembrane signaling receptor activity', 'MHC class II receptor activity', 'C-C chemokine receptor activity' and 'peptide antigen binding activity'. A total of 10 significant KEGG pathway terms were also identified including 'cell adhesion molecules (CAMs)' and 'cytokine-cytokine receptor interaction'.

Validation in the GEO database. To confirm that the prognostic genes identified using TCGA analysis were also important in other LUAD cases, two independent eligible cohorts of LUAD cases from the GEO database (accession numbers GES37745 and GES29013) were selected for validation, and the calculated OS rate of genes was performed the GEO database $(\mathrm{P}<0.05 ; \mathrm{HR}<1$; Table SIV). Only 4 genes were validated as positively associated with prognosis. They were GTPase IMAP family member 1 (GIMAP1), T-cell surface glycoprotein CD1b (CD1B), ITGAL and leukocyte surface antigen CD53 (CD53). The HR value of these 4 genes in the GSE37745 dataset were as follows: GIMAP1, $0.51(\mathrm{P}=0.01)$; CD1B, $0.51(\mathrm{P}=0.01)$; ITGAL, $0.58(\mathrm{P}=0.01)$ and $\mathrm{CD} 53,0.63$ 


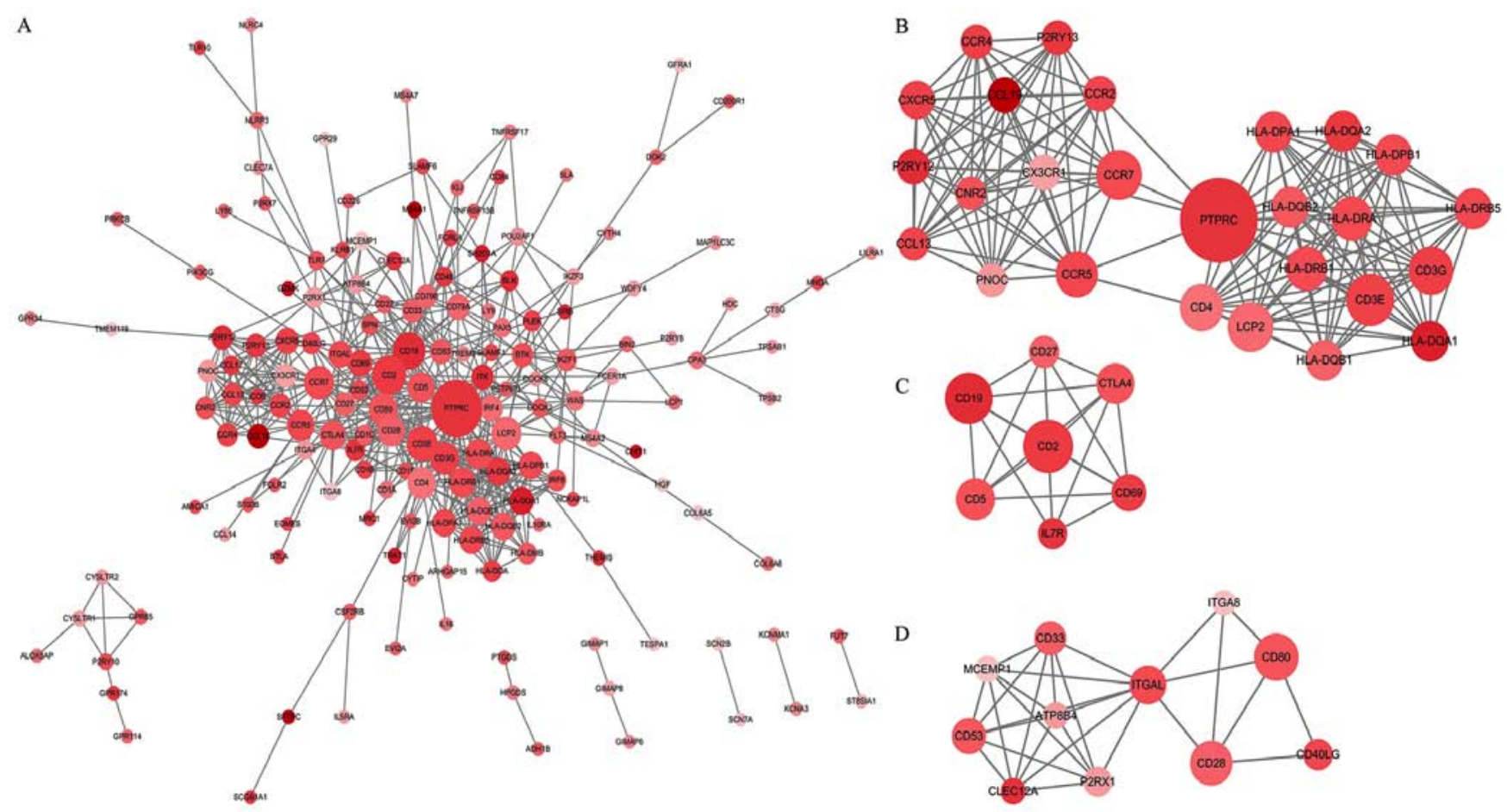

Figure 4. PPI network construction of 281 downregulated DEGs of prognostic value. (A) PPI network of the 281 downregulated DEGs constructed with Cytoscape software. (B-D) Top three modules of the PPI network. The color of a node in the PPI network reflects the log fold change value based on immune scores, and the size of the node indicates the number of proteins interacting with the designated protein based on immune scores. PPI, protein-protein interaction.

A

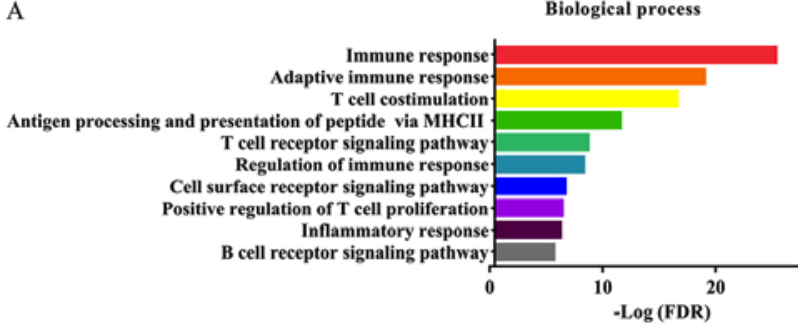

$\mathrm{C}$

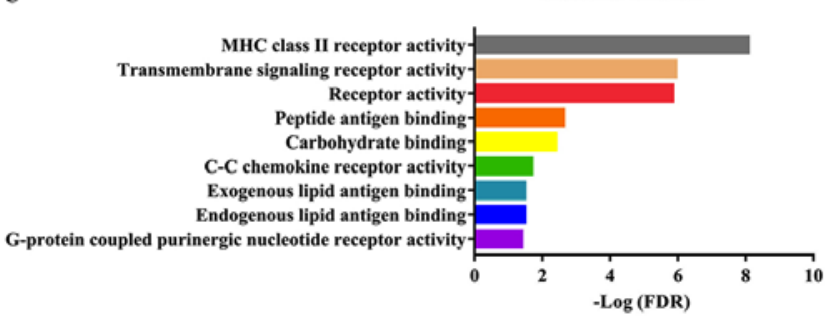

B

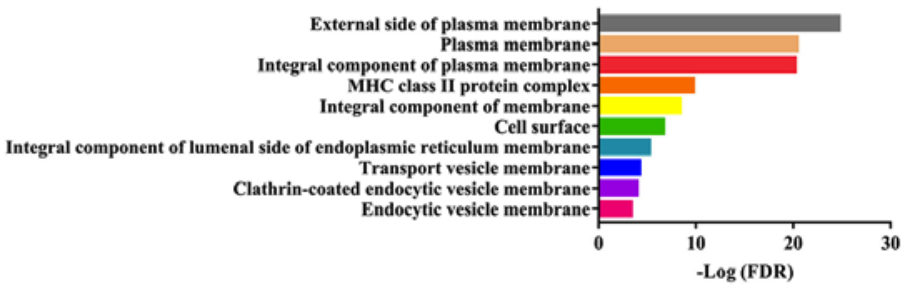

D

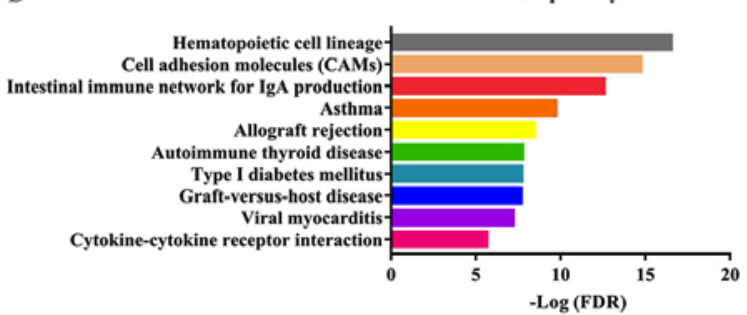

Figure 5. Functional analysis of genes of prognostic value. (A) Biological process, (B) Cellular component and (C) Molecular function of Gene Ontology terms. (D) KEGG pathway analysis. KEGG, Kyoto Encyclopedia of Genes and Genomes; FDR, false discovery rate.

$(\mathrm{P}=0.02)$. The HR value of the 4 aforementioned genes in the GSE29013 dataset were as follows: GIMAP1, $0.50(\mathrm{P}=0.02)$; CD1B, 0.51 ( $\mathrm{P}=0.04)$; ITGAL, $0.71(\mathrm{P}=0.01)$ and CD53, 0.54 $(\mathrm{P}=0.04)$. Although other genes were associated with prognosis in the TCGA database, they were not associated with prognosis from the GEO database. As a result, GIMAP1, CD1B, ITGAL and CD53 were validated as positively associated with prognosis and downregulation of these 4 genes was associated with poor prognosis (Fig. 6).

\section{Discussion}

A number of different studies have applied the ESTIMATE algorithm to breast, prostate and colon cancer, indicating that it was a useful and effective tool for analyzing large datasets (29-31). Previous studies also applied this algorithm to mining TME-related genes and identified high number of novel genes that were associated with prognosis in glioblastoma and cutaneous melanoma $(32,33)$. To the best of our knowledge, 

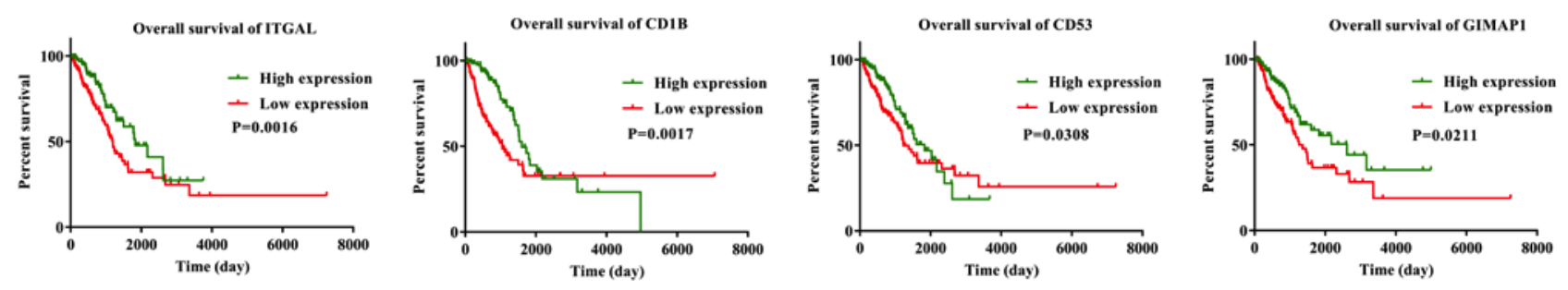

Figure 6. Validation of The Cancer Genome Atlas results in two lung adenocarcinoma cohorts from the Gene Expression Omnibus database. Kaplan-Meier survival curves for the verified genes.

the current study is the first study where the ESTIMATE scoring algorithm was used to identify tumor-related genes that were associated with the OS of patients with LUAD in the TCGA database. A total of 281 prognosis-related genes were identified and were found to be involved in the immune and inflammatory response and were subsequently validated in two LUAD cohorts from the GEO database. Finally, a total of 4 prognosis-related genes were associated with OS in the TCGA and GEO database.

Firstly, patients with stage III-IV TNM LUAD had lower immune and stromal scores compared with those with stage I-II TNM LUAD. Patients with distant metastasis had lower immune and stromal scores compared with those without distant metastasis. The Kaplan-Meier survival curves revealed that patients with low immune/stromal scores had a poorer prognosis compared with those with high immune/stromal scores. All of the aforementioned results suggested that high immune/stromal scores were associated with the prognosis of patients with LUAD. This is consistent with previous reports that the inflamed and immunogenic TME was associated with favorable patient survival in lung adenocarcinoma $(15,34)$.

Secondly, downregulated DEGs were identified from the comparison of low vs. high immune score groups, and also from the comparison of low vs. high stromal score groups. Subsequently, 519 DEGs that were downregulated in both the immune and the stromal score groups were analyzed further. GO and KEGG pathway analysis of these genes revealed that the majority were related to immune response, inflammatory response, $\mathrm{T}$ cell co-stimulation and chemokine-mediated signaling pathway. It is noteworthy that Staphylococcus aureus infection was ranked first in the KEGG pathway analysis. Pulmonary bacterial infections are frequently found in advanced stages of lung cancer (35). Hattar et al (36) also reported that purified lipoteichoic acids of $S$. aureus could induce growth of lung adenocarcinoma cell lines and these effects were mediated by inflammatory mediators such as ligation of Toll-like receptor 2 and interleukin (IL)-8. Thus, infections with Gram-positive bacteria might cause persistent inflammation and activated inflammatory cascades, which contribute to tumor growth in lung cancer. The results of GO and KEGG pathway analysis indicated that a number of immune cells, chemokines, cytokines and extracellular matrix components could be involved in regulating the relationship between LUAD and TME (11).

Thirdly, the prognostic value of the 519 DEGs was assessed and 281 DEGS were associated with OS in patients with LUAD. GO and KEGG pathway analysis of the 281 genes of prognostic value was performed, majority of which were related to immune and inflammatory response (Fig. 5A). A PPI network of the 281 genes was created and the top three modules were selected using a MCODE plugin. There was high connectivity between the nodes in these modules, including CCR7, HLA-DQB1 and CTLA4, which have been reported to be associated with regulating immune and inflammatory responses and OS of patients with LUAD (3,37-39).

Finally, two independent LUAD cohorts from GEO database were used to validate the prognostic value of genes identified in TCGA database. Only 4 genes, GIMAP1, CD1B, ITGAL and CD53, were found to have prognostic value in the TCGA database and 2 datasets from the GEO database. GIMAP1 belongs to the family of guanosine triphosphatases of the immunity-associated proteins (GIMAPs) (40). Previous studies have shown that the deregulated expression of GIMAP genes is associated with lymphomas (41). GIMAP1 is required for the establishment and maintenance of B cell survival and mature B cells do not survive without GIMAP1 expression (42). CD1B is a member of the CD1 molecule family which specializes in presenting lipid antigens to $T$ cells (43). The activation of CD1 restricts $T$ cells in vivo leading to rapid antitumor cytotoxicity and interferon- $\gamma$ production, which could prevent tumor metastasis (44). Bagchi et al (45) reported that CD1B-autoreactive $T$ cells could recognize phospholipid antigens and exert antitumor immunity against $\mathrm{CD}^{+} \mathrm{B}^{+} \mathrm{T}$ cell lymphoma. ITGAL, which is also known as LFA-1 and CD11a, is a member of the integrins family and is expressed on all leukocytes. LFA-1 is associated with myeloid cell function in the TME and plays an important role in the function of regulatory $\mathrm{T}$ (T-reg) cells that infiltrate tumors. A previous study indicated that neutrophils were recruited into the tumor via increased expression of LFA-1, activated by estradiol and transforming growth factor $\beta 1$ in a mouse model of estrogen receptor-positive breast cancer (46). In colon cancer, another study reported that IL-18 was involved in eosinophil-mediated antitumor activity by upregulating LFA-1 and ICAM-1 (47). LFA-1 is also necessary for the development and function of T-reg cells and knockdown of LFA-1 leads to an increase in autoimmunity (48). CD53 is a member of the tetraspanin family and participates in the formation of a complex with integrins on the plasma membrane (49). It has been reported that CD53 is associated with asthma risk in the general population and recurrent bacteria, fungi and viruses infections $(50,51)$; however, the relationship between CD53 and cancer has rarely been reported. Through reviewing the literature, these 4 genes were found to be immune/inflammation-related genes; 
however, the relationship between these 4 genes and lung cancer has also been rarely reported. In the present study, downregulation of these 4 genes was associated with a worse OS in patients with LUAD, therefore, they have potential to become novel cancer biomarkers.

There are some limitations to the present study, which are noteworthy. Firstly, the exploration occurred at a bioinformatics level, thus further experiments are required to validate the exact mechanism of these 4 genes in vitro and in vivo. Secondly, patients with LUAD in TCGA database received different treatments, such as chemotherapy, targeted molecular therapy or immunotherapy. These treatments could affect gene expression and OS rates of patients. Thus, clinical potential of these 4 genes requires a larger sample size to be confirmed.

In conclusion, using the ESTIMATE algorithm to calculate the immune and stromal scores of patients with LUAD in TCGA database, it was found that low immune/stromal scores were associated with a worse TNM stage, a greater likelihood of distant metastasis and an unfavorable overall survival in patients with LUAD. TME-related genes were identified and 4 of them were validated in two independent LUAD cohorts from GEO database. These 4 genes were GIMAP1, CD1B, ITGAL and CD53, and were strongly associated with immune response and inflammatory response. The present study has obtained an improved comprehensive understanding of TME by mining TCGA database and identified 4 immune/inflammatory-related genes, which have the potential to predict prognosis of patients with LUAD.

\section{Acknowledgements}

Not applicable.

\section{Funding}

The present study was funded by the Fundamental Science Research Project of Xi'an Jiaotong University (grant no. 1191329849) and National Natural Science Foundation of China (grant no. 81672300).

\section{Availability of data and materials}

The datasets analyzed during the present study are available in The Cancer Genome Atlas repository (https://cancergenome.nih.gov/) and the GEO repository (https://www.ncbi.nlm.nih.gov/geo/).

\section{Authors' contributions}

JY designed the study and wrote the manuscript. BY, LZ and BXL contributed to the acquisition of data. YC, XM and RYS performed the bioinformatics analysis. XL and WW analyzed data. SY contributed to the experimental design and was responsible for revising the manuscript and approving the version to be published. All authors read and approved the final version of the manuscript.

\section{Ethics approval and consent to participate}

Not applicable.

\section{Patient consent for publication}

Not applicable.

\section{Competing interests}

The authors declare that they have no competing interests.

\section{References}

1. Travis WD, Brambilla E, Nicholson AG, Yatabe Y, Austin JHM Beasley MB, Chirieac LR, Dacic S, Duhig E, Flieder DB, et al: The 2015 World Health Organization classification of lung tumors: Impact of genetic, clinical and radiologic advances since the 2004 classification. J Thorac Oncol 10: 1243-1260, 2015.

2. Osmani L, Askin F, Gabrielson E and Li QK: Current WHO guidelines and the critical role of immunohistochemical markers in the subclassification of non-small cell lung carcinoma (NSCLC): Moving from targeted therapy to immunotherapy. Semin Cancer Biol 52: 103-109, 2018.

3. Reck M, Bondarenko I, Luft A, Serwatowski P, Barlesi F, Chacko R, Sebastian M, Lu H, Cuillerot JM and Lynch TJ: Ipilimumab in combination with paclitaxel and carboplatin as first-line therapy in extensive-disease-small-cell lung cancer: Results from a randomized, double-blind, multicenter phase 2 trial. Ann Oncol 24: 75-83, 2013.

4. Horinouchi H, Nishio M, Hida T, Nakagawa K, Sakai H, Nogami N, Atagi S, Takahashi T, Saka H, Takenoyama M, et al: Three-year follow-up results from phase II studies of nivolumab in Japanese patients with previously treated advanced non-small cell lung cancer: Pooled analysis of ONO-4538-05 and ONO-4538-06 studies. Cancer Med 8: 5183-5193, 2019.

5. Brahmer JR, Tykodi SS, Chow LQ, Hwu WJ, Topalian SL, Hwu P, Drake CG, Camacho LH, Kauh J, Odunsi K, et al: Safety and activity of anti-PD-L1 antibody in patients with advanced cancer. N Engl J Med 366: 2455-2465, 2012.

6. Hellmann MD, Rizvi NA, Goldman JW, Gettinger SN, Borghaei H, Brahmer JR, Ready NE, Gerber DE, Chow LQ, Juergens RA, et al: Nivolumab plus ipilimumab as first-line treatment for advanced non-small-cell lung cancer (CheckMate 012): Results of an open-label, phase 1, multicohort study. Lancet Oncol 18: 31-41, 2017.

7. Fukumura D, Kloepper J, Amoozgar Z, Duda DG and Jain RK: Enhancing cancer immunotherapy using antiangiogenics: Opportunities and challenges. Nat Rev Clin Oncol 15: 325-340, 2018.

8. Datta M, Coussens LM, Nishikawa H, Hodi FS and Jain RK: Reprogramming the tumor microenvironment to improve immunotherapy: Emerging strategies and combination therapies. Am Soc Clin Oncol Educ Book 39: 165-174, 2019.

9. Li HY, McSharry M, Bullock B, Nguyen TT, Kwak J, Poczobutt JM, Sippel TR, Heasley LE, Weiser-Evans MC, Clambey ET and Nemenoff RA: The tumor microenvironment regulates sensitivity of murine lung tumors to PD-1/PD-L1 antibody blockade. Cancer Immunol Res 5: 767-777, 2017.

10. Gao D and Mittal V: The role of bone-marrow-derived cells in tumor growth, metastasis initiation and progression. Trends Mol Med 15: 333-343, 2009.

11. Mittal V, El Rayes T, Narula N, McGraw TE, Altorki NK and Barcellos-Hoff MH: The microenvironment of lung cancer and therapeutic implications. Adv Exp Med Biol 890: 75-110, 2016.

12. Binnewies M, Roberts EW, Kersten K, Chan V, Fearon DF, Merad M, Coussens LM, Gabrilovich DI, Ostrand-Rosenberg S, Hedrick CC, et al: Understanding the tumor immune microenvironment (TIME) for effective therapy. Nat Med 24: 541-550, 2018.

13. Rhee JK, Jung YC, Kim KR, Yoo J, Kim J, Lee YJ, Ko YH, Lee HH, Cho BC and Kim TM: Impact of tumor purity on immune gene expression and clustering analyses across multiple cancer types. Cancer Immunol Res 6: 87-97, 2018.

14. Pages F, Galon J, Dieu-Nosjean MC, Tartour E, Sautes-Fridman C and Fridman WH: Immune infiltration in human tumors: A prognostic factor that should not be ignored. Oncogene 29: 1093-1102, 2010.

15. Givechian KB, Garner C, Benz S, Song B, Rabizadeh S and Soon-Shiong P: An immunogenic NSCLC microenvironment is associated with favorable survival in lung adenocarcinoma. Oncotarget 10: 1840-1849, 2019. 
16. Patel SJ, Sanjana NE, Kishton RJ, Eidizadeh A, Vodnala SK, Cam M, Gartner JJ, Jia L, Steinberg SM, Yamamoto TN, et al: Identification of essential genes for cancer immunotherapy. Nature 548: 537-542, 2017.

17. Brown SD, Warren RL, Gibb EA, Martin SD, Spinelli JJ, Nelson BH and Holt RA: Neo-antigens predicted by tumor genome meta-analysis correlate with increased patient survival. Genome Res 24: 743-750, 2014.

18. Lyssiotis CA and Kimmelman AC: Metabolic interactions in the tumor microenvironment. Trends Cell Biol 27: 863-875, 2017.

19. Newman AM, Liu CL, Green MR, Gentles AJ, Feng W, Xu Y, Hoang CD, Diehn M and Alizadeh AA: Robust enumeration of cell subsets from tissue expression profiles. Nat Methods 12: 453-457, 2015.

20. Yoshihara K, Shahmoradgoli M, Martinez E, Vegesna R, Kim H, Torres-Garcia W, Treviño V, Shen H, Laird PW, Levine DA, et al: Inferring tumour purity and stromal and immune cell admixture from expression data. Nat Commun 4: 2612, 2013.

21. Botling J, Edlund K, Lohr M, Hellwig B, Holmberg L, Lambe M, Berglund A, Ekman S, Bergqvist M, Pontén F, et al: Biomarker discovery in non-small cell lung cancer: Integrating gene expression profiling, meta-analysis, and tissue microarray validation. Clin Cancer Res 19: 194-204, 2013.

22. Xie Y, Xiao G, Coombes KR, Behrens C, Solis LM, Raso G, Girard L, Erickson HS, Roth J, Heymach JV, et al: Robust gene expression signature from formalin-fixed paraffin-embedded samples predicts prognosis of non-small-cell lung cancer patients. Clin Cancer Res 17: 5705-5714, 2011.

23. Ritchie ME, Phipson B, Wu D, Hu Y, Law CW, Shi W and Smyth GK: limma powers differential expression analyses for RNA-sequencing and microarray studies. Nucleic Acids Res 43: e47, 2015

24. Ito K and Murphy D: Application of ggplot2 to pharmacometric graphics. CPT Pharmacometrics Syst Pharmacol 2: e79, 2013.

25. Szklarczyk D, Franceschini A, Wyder S, Forslund K, Heller D, Huerta-Cepas J, Simonovic M, Roth A, Santos A, Tsafou KP, et al: STRING v10: Protein-protein interaction networks, integrated over the tree of life. Nucleic Acids Res 43: D447-D452, 2015.

26. Shannon P, Markiel A, Ozier O, Baliga NS, Wang JT, Ramage D, Amin N, Schwikowski B and Ideker T: Cytoscape: A software environment for integrated models of biomolecular interaction networks. Genome Res 13: 2498-2504, 2003.

27. Huang da W, Sherman BT and Lempicki RA: Systematic and integrative analysis of large gene lists using DAVID bioinformatics resources. Nat Protoc 4: 44-57, 2009.

28. Tokumo M, Toyooka S, Kiura K, Shigematsu H, Tomii K, Aoe M, Ichimura K, Tsuda T, Yano M, Tsukuda K, et al: The relationship between epidermal growth factor receptor mutations and clinicopathologic features in non-small cell lung cancers. Clin Cancer Res 11: 1167-1173, 2005.

29. Priedigkeit N, Watters RJ, Lucas PC, Basudan A, Bhargava R, Horne W, Kolls JK, Fang Z, Rosenzweig MQ, Brufsky AM, et al: Exome-capture RNA sequencing of decade-old breast cancers and matched decalcified bone metastases. JCI Insight 2: 95703, 2017.

30. Shah N, Wang P, Wongvipat J, Karthaus WR, Abida W, Armenia J, Rockowitz S, Drier Y, Bernstein BE, Long HW, et al: Regulation of the glucocorticoid receptor via a BET-dependent enhancer drives antiandrogen resistance in prostate cancer. ELife 6: e27861, 2017.

31. Alonso MH, Ausso S, Lopez-Doriga A, Cordero D, Guinó E, Solé X, Barenys M, de Oca J, Capella G, Salazar R, et al: Comprehensive analysis of copy number aberrations in microsatellite stable colon cancer in view of stromal component. $\mathrm{Br} \mathbf{J}$ Cancer 117: 421-431, 2017.

32. Jia D, Li S, Li D, Xue H, Yang D and Liu Y: Mining TCGA database for genes of prognostic value in glioblastoma microenvironment. Aging (Albany NY) 10: 592-605, 2018.

33. Yang S, Liu T, Nan H, Wang Y, Chen H, Zhang X, Zhang Y, Shen B, Qian P, Xu S, et al: Comprehensive analysis of prognostic immune-related genes in the tumor microenvironment of cutaneous melanoma. J Cell Physiol 235: 1025-1035, 2020.

34. Al-Shibli KI, Donnem T, Al-Saad S, Persson M, Bremnes RM and Busund LT: Prognostic effect of epithelial and stromal lymphocyte infiltration in non-small cell lung cancer. Clin Cancer Res 14: 5220-5227, 2008.
35. Berghmans T, Sculier JP and Klastersky J: A prospective study of infections in lung cancer patients admitted to the hospital. Chest 124: 114-120, 2003.

36. Hattar K, Reinert CP, Sibelius U, Gökyildirim MY, Subtil FSB, Wilhelm J, Eul B, Dahlem G, Grimminger F, Seeger W and Grandel U: Lipoteichoic acids from staphylococcus aureus stimulate proliferation of human non-small-cell lung cancer cells in vitro. Cancer Immunol Immunother 66: 799-809, 2017.

37. Zhang L, Li M, Deng B, Dai N, Feng Y, Shan J, Yang Y, Mao C, Huang P, Xu C and Wang D: HLA-DQB1 expression on tumor cells is a novel favorable prognostic factor for relapse in early-stage lung adenocarcinoma. Cancer Manag Res 11: 2605-2616, 2019

38. Itakura M, Terashima Y, Shingyoji M, Yokoi S, Ohira M, Kageyama H, Matui Y, Yoshida Y, Ashinuma H, Moriya Y, et al: High CC chemokine receptor 7 expression improves postoperative prognosis of lung adenocarcinoma patients. Br J Cancer 109: 1100-1108, 2013.

39. Leach DR, Krummel MF and Allison JP: Enhancement of antitumor immunity by CTLA-4 blockade. Science 271: 1734-1736, 1996.

40. Krucken J, Stamm O, Schmitt-Wrede HP, Mincheva A, Lichter P and Wunderlich F: Spleen-specific expression of the malaria-inducible intronless mouse gene imap38. J Biol Chem 274: 24383-24391, 1999.

41. Chadwick N,Zeef L, Portillo V, Boros J, Hoyle S, van Doesburg JC and Buckle AM: Notch protection against apoptosis in T-ALL cells mediated by GIMAP5. Blood Cells Mol Dis 45: 201-209, 2010.

42. Webb LM, Datta P, Bell SE, Kitamura D, Turner M and Butcher GW: GIMAP1 Is essential for the survival of naive and activated B cells in vivo. J Immunol 196: 207-216, 2016.

43. Brigl M and Brenner MB: CD1: Antigen presentation and T cell function. Annu Rev Immunol 22: 817-890, 2004.

44. HayakawaY,GodfreyDIandSmythMJ:Alpha-galactosylceramide: Potential immunomodulatory activity and future application. Curr Med Chem 11: 241-252, 2004.

45. Bagchi S, Li S and Wang CR: CD1b-autoreactive T cells recognize phospholipid antigens and contribute to antitumor immunity against a CD1b(+) T cell lymphoma. Oncoimmunology 5: e1213932, 2016.

46. Vazquez Rodriguez G, Abrahamsson A, Jensen LD and Dabrosin C: Estradiol promotes breast cancer cell migration via recruitment and activation of neutrophils. Cancer Immunol Res 5: 234-247, 2017.

47. Gatault S, Delbeke M, Driss V, Sarazin A, Dendooven A, Kahn JE, Lefèvre G and Capron M: IL-18 is involved in eosinophil-mediated tumoricidal activity against a colon carcinoma cell line by upregulating LFA-1 and ICAM-1. J Immunol 195: 2483-2492, 2015.

48. Wohler J, Bullard D, Schoeb T and Barnum S: LFA-1 is critical for regulatory T cell homeostasis and function. Mol Immunol 46: 2424-2428, 2009.

49. Mannion BA, Berditchevski F, Kraeft SK, Chen LB and Hemler ME: Transmembrane-4 superfamily proteins CD81 (TAPA-1), CD82, CD63, and CD53 specifically associated with integrin alpha 4 beta 1 (CD49d/CD29). J Immunol 157: 2039-2047, 1996.

50. Lee H, Bae S, Jang J, Choi BW, Park CS, Park JS, Lee SH and Yoon Y: CD53, a suppressor of inflammatory cytokine production, is associated with population asthma risk via the functional promoter polymorphism $-1560 \mathrm{C}>\mathrm{T}$. Biochim Biophys Acta 1830: 3011-3018, 2013.

51. Mollinedo F, Fontan G, Barasoain I and Lazo PA: Recurrent infectious diseases in human CD53 deficiency. Clin Diagn Lab Immunol 4: 229-231, 1997.

This work is licensed under a Creative Commons Attribution-NonCommercial-NoDerivatives 4.0 International (CC BY-NC-ND 4.0) License. 